\title{
Enhanced photoelectric detection of NV magnetic resonances in diamond under dual-beam excitation
}

\author{
E. Bourgeois, ${ }^{1,2, *}$ E. Londero, ${ }^{3}$ K. Buczak, ${ }^{4}$ J. Hruby, ${ }^{2}$ M. Gulka, ${ }^{2}$ Y. Balasubramaniam, ${ }^{2}$ G. Wachter,${ }^{4}$ J. Stursa, ${ }^{5}$ K. Dobes, ${ }^{6}$ \\ F. Aumayr, ${ }^{6}$ M. Trupke, ${ }^{4}$ A. Gali, ${ }^{3,7}$ and M. Nesladek ${ }^{1,2, \dagger}$ \\ ${ }^{1}$ IMOMEC Division, IMEC, Wetenschapspark 1, B-3590 Diepenbeek, Belgium \\ ${ }^{2}$ Institute for Materials Research (IMO), Hasselt University, Wetenschapspark 1, B-3590 Diepenbeek, Belgium \\ ${ }^{3}$ Institute for Solid State Physics and Optics, Wigner Research Centre for Physics, Hungarian Academy of Sciences, \\ PO Box 49, H-1525 Budapest, Hungary \\ ${ }^{4}$ Vienna Center for Quantum Science and Technology, Atominstitut, TU Wien, A-1020 Vienna, Austria \\ ${ }^{5}$ Nuclear Physics Institute, v.v.i., ASCR, CZ-25068 Rez, Czech Republic \\ ${ }^{6}$ Institute of Applied Physics, TU Wien, Wiedner Hauptstrasse 8-10, A-1040 Vienna, Austria \\ ${ }^{7}$ Department of Atomic Physics, Budapest University of Technology and Economics, Budafoki út 8, H-1111 Budapest, Hungary
}

(Received 8 July 2016; revised manuscript received 9 November 2016; published 17 January 2017)

\begin{abstract}
The core issue for the implementation of NV center qubit technology is a sensitive readout of the NV spin state. We present here a detailed theoretical and experimental study of NV center photoionization processes, used as a basis for the design of a dual-beam photoelectric method for the detection of NV magnetic resonances (PDMR). This scheme, based on NV one-photon ionization, is significantly more efficient than the previously reported single-beam excitation scheme. We demonstrate this technique on small ensembles of $\sim 10$ shallow NVs implanted in electronic grade diamond (a relevant material for quantum technology), on which we achieve a cw magnetic resonance contrast of $9 \%$-three times enhanced compared to previous work. The dual-beam PDMR scheme allows independent control of the photoionization rate and spin magnetic resonance contrast. Under a similar excitation, we obtain a significantly higher photocurrent, and thus an improved signal-to-noise ratio, compared to single-beam PDMR. Finally, this scheme is predicted to enhance magnetic resonance contrast in the case of samples with a high proportion of substitutional nitrogen defects, and could therefore enable the photoelectric readout of single NV spins.
\end{abstract}

DOI: 10.1103/PhysRevB.95.041402

The negatively charged nitrogen-vacancy $\left(\mathrm{NV}^{-}\right)$center in diamond has attracted particular attention as a room temperature solid-state qubit [1] that can be read out by optical detection of magnetic resonances (ODMR) [2]. Numerous applications in the field of solid-state quantum information processing [3] and sensing [4-10] are being studied.

We have recently developed a method for the photoelectric detection of $\mathrm{NV}^{-}$electron spin magnetic resonances (PDMR) [11], performed directly on a diamond chip equipped with electric contacts and based on the electric detection of charge carriers promoted to the diamond conduction band (CB) by two-photon ionization of $\mathrm{NV}^{-}$under green illumination (single-beam PDMR, or s-PDMR) (Fig. 1). The compatibility of this readout method with coherent manipulations of $\mathrm{NV}$ spin ensembles was recently reported [12]. Enhancing the photocurrent signal and MR contrast is critical to reach a high sensitivity of magnetic resonance (MR) detection $[4,13]$ and achieve a photoelectric readout of single NV spins, essential for quantum applications. Using s-PDMR, we obtained a detection rate of $\sim 10^{7}$ electrons $\mathrm{s}^{-1}$ per $\mathrm{NV}^{-}$(numerical aperture of the objective: 0.95; green illumination: $3.4 \mathrm{~mW}$; electric field: $2.4 \times 10^{4} \mathrm{~V} \mathrm{~cm}^{-1}$ ), compared to $2 \times 10^{4}$ photons s$^{-1}$ per $\mathrm{NV}^{-}$using confocal ODMR in the same conditions. However, the ionization of single substitutional nitrogen $\left(\mathrm{N}_{\mathrm{s}}^{0}\right)$ is one of the factors limiting the s-PDMR contrast [11].

\footnotetext{
*emilie.bourgeois@uhasselt.be

†milos.nesladek@uhasselt.be
}

To explore the photophysics behind the PDMR scheme and optimize its performances, we performed $a b$ initio calculations of $\mathrm{N}_{\mathrm{s}}^{0}, \mathrm{NV}^{-}$, and $\mathrm{NV}^{0}$ ionization cross sections, and compared the results to experimental characterizations of the ionization bands. In this way we demonstrate that under blue illumination, the ionization of $\mathrm{NV}^{-}$can be achieved by a more effective one-photon process, leading to a higher photocurrent-and therefore a higher signal-to-noise $(\mathrm{S} / \mathrm{N})$ ratio-than green illumination of identical power. Based on this result, we designed a dual-beam PDMR (d-PDMR) scheme (Fig. 1), in which pulsed blue light directly ionizes $\mathrm{NV}^{-}$and converts the resultant $\mathrm{NV}^{0}$ back to $\mathrm{NV}^{-}$by one-photon processes, while simultaneous cw green illumination independently controls the MR contrast. We validated this scheme on small ensembles of $\sim 10$ shallow $\mathrm{NV}^{-}$centers implanted in electronic grade diamond, which represents a downscaling of the photoelectric detection by a factor $\sim 10^{5}$ compared to a previous publication [11]. The d-PDMR scheme leads to enhanced MR contrast under low power illumination and is anticipated to result in increased MR contrast in the case of samples with a high $\left[\mathrm{N}_{\mathrm{s}}^{0}\right] /\left[\mathrm{NV}^{-}\right]$ratio. It could therefore hold promise for a photoelectric readout of single $\mathrm{NV}^{-}$spins, since the proportion of $\mathrm{N}_{\mathrm{s}}^{0}$ in the detection volume remains substantial even in the case of single $\mathrm{NV}^{-}$contained in ultrapure diamond.

For the density functional theory (DFT) calculation of photoionization cross sections [14], we consider the photoionization probability as directly proportional to the absorption cross section, which depends on the imaginary part of the dielectric function related to the transition between the initial 


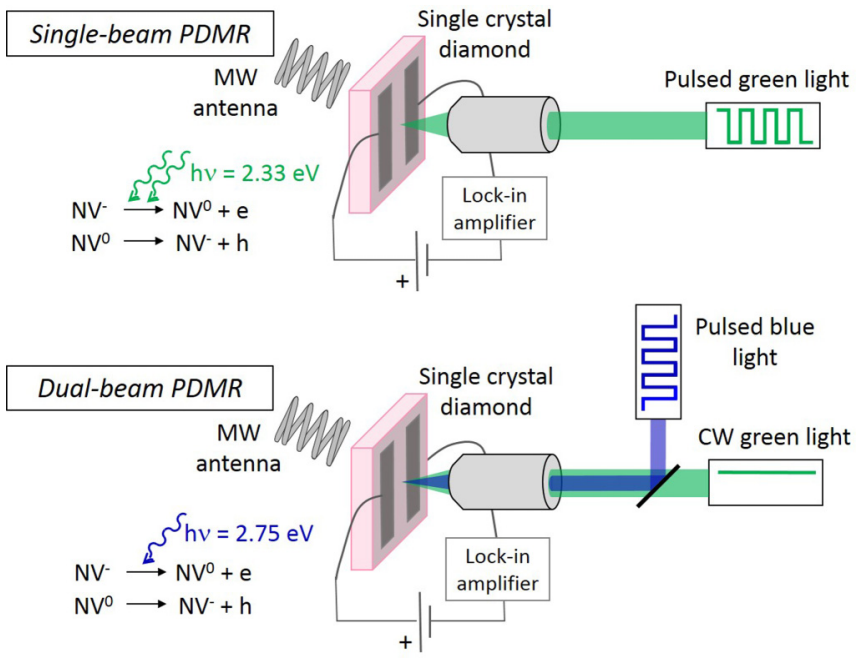

FIG. 1. Schematic diagram of the s-PDMR and d-PDMR schemes. For s-PDMR, pulsed green light ionizes $\mathrm{NV}^{-}$and converts $\mathrm{NV}^{0}$ back to $\mathrm{NV}^{-}$by two-photon processes. For d-PDMR, pulsed blue light directly ionizes $\mathrm{NV}^{-}$and converts $\mathrm{NV}^{0}$ back to $\mathrm{NV}^{-}$ by one-photon processes, while $\mathrm{cw}$ green light induces shelving transitions to the metastable state.

ground state and the final excited state. This transition is well approximated by the transition of a single electron from/to the in-gap defect level to/from the band edges. The imaginary part of the dielectric function can thus be calculated between the corresponding Kohn-Sham levels. We calculate the lowest excitation energy that corresponds to the pure electronic transition [zero-phonon line (ZPL) energy] by the constraint DFT approach. Based on our previous studies $[15,16]$, we use a range-separated and screened hybrid density functional HSE06 [17,18]. Optical transitions to the bands require an accurate calculation of the electron density of states. We therefore apply a generalized gradient approximated PerdewBurke-Ernzerhof (PBE) functional to calculate the optical transition dipole moments [19]. The defects are modeled in a 512-atom diamond supercell.

For PDMR measurements, we used an electronic grade type-IIa diamond implanted with ${ }^{14} \mathrm{~N}^{4+}$ ions (sample TP4), resulting after annealing in the formation of a shallow $\mathrm{NV}^{-}$layer (density, $\sim 30 \mu \mathrm{m}^{-2}$; depth, $12 \pm 4 \mathrm{~nm}$ ). For photocurrent spectroscopy, an as-received type-Ib diamond plate (sample R) was used as a reference, while two others were respectively proton irradiated (sample $\mathrm{A},\left[\mathrm{NV}^{-}\right] \sim 35 \mathrm{ppm}$ ) and electron irradiated (sample E2, $\left[\mathrm{NV}^{-}\right] \sim 20 \mathrm{ppm}$ ) and annealed. Coplanar electrodes were prepared on the surface of these diamond plates. The type-Ib samples were characterized by photocurrent, photoluminescence, Fourier transform infrared (FTIR), and UV-visible absorption spectroscopy (see note 1 in the Supplemental Material [14]).

To realize the d-PDMR scheme, a dc electric field $(2.4 \times$ $\left.10^{4} \mathrm{~V} \mathrm{~cm}^{-1}\right)$ is applied in between electrodes. A collimated blue $(2.75 \mathrm{eV})$ laser beam, pulsed at $131 \mathrm{~Hz}$, is focused in between the electrodes onto the diamond surface, resulting in a light spot diameter of $\sim 580 \mathrm{~nm}$. Small ensembles of eight to ten NV centers are thus located in the focus of the objective. A cw green $(2.33 \mathrm{eV})$ laser light is combined

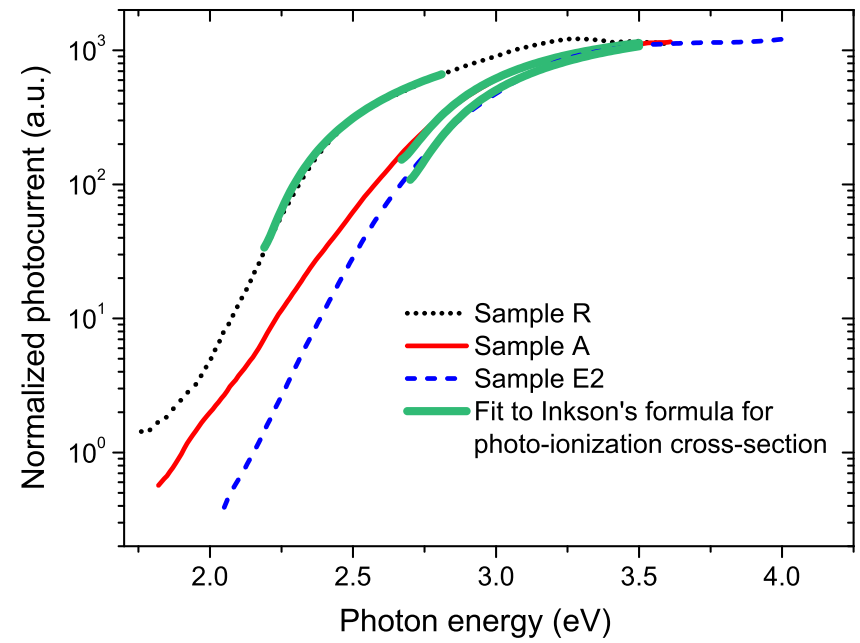

FIG. 2. Measured photoionization bands. Comparison between photocurrent spectra measured on reference type-Ib diamond [sample $\left.\mathrm{R}, E_{\mathrm{i}}=2.18(3) \mathrm{eV}\right]$ and irradiated and annealed type-Ib diamonds [sample A, $E_{\mathrm{i}}=2.66(4) \mathrm{eV}$; sample E2, $E_{\mathrm{i}}=2.69(3) \mathrm{eV}$ ]. $E_{\mathrm{i}}$ : Threshold ionization energy from Inkson's fitting.

with the blue beam using the same objective. The resulting photocurrent is preamplified and measured using a lock-in amplifier referenced to the blue light pulsing frequency, so that the photocurrent induced by the $\mathrm{cw}$ green light does not contribute to the measured signal. The diamond chip is mounted on a circuit board equipped with microwave antennas [20].

To get insight into the photoionization processes involved in PDMR, we first studied experimentally the energy dependence of $\mathrm{NV}$ and $\mathrm{N}_{\mathrm{s}}^{0}$ photoionization cross sections by photocurrent spectroscopy. The understanding of optical transitions is important for resolving photorecombination phenomena [21]. These measurements were performed on type-Ib diamonds, since a high density of defects is necessary to reach a high dynamic range of detection, required for the precise determination of the photoionization thresholds. The photocurrent spectrum measured on a type-Ib reference diamond (sample $\left.\mathrm{R},\left[\mathrm{N}_{\mathrm{s}}^{0}\right] \sim 160 \mathrm{ppm}\right)$ displays a photoionization band with a threshold ionization energy of $\sim 2.2 \mathrm{eV}$ (Fig. 2), obtained by fitting experimental data to Inkson's formula for the photoionization cross section of deep defects [22]. This band corresponds to the ionization of $\mathrm{N}_{\mathrm{s}}^{0}$ to $\mathrm{N}_{\mathrm{s}}^{+}[23,24]$. Compared to nonirradiated diamond, the photocurrent spectra measured on proton- and electron-irradiated type-Ib diamonds (in which $\sim 10 \%$ of $\mathrm{N}_{\mathrm{s}}$ defects are converted to $\mathrm{NV}^{-}$centers) show a blueshift and the formation of an ionization band with a threshold at $\sim 2.7 \mathrm{eV}$ (Fig. 2). Photoluminescence, FTIR, and optical absorption spectroscopy indicate that the dominant defects in these samples are $\mathrm{N}_{\mathrm{s}}$ and $\mathrm{NV}$, with some additional spurious defects (possibly associated to $\mathrm{Ni}$ ) in sample A (see note 2 in the Supplemental Material).

We used $a b$ initio modeling to determine the ionization cross sections of $\mathrm{N}_{\mathrm{s}}$ and $\mathrm{NV}$ defects as a function of the photoexcitation energy [Fig. 3(a)]. In the case of $\mathrm{N}_{\mathrm{s}}^{0}$, an $a_{1}$ orbital resides in the gap (see Supplemental Fig. 1), from which the electron may be promoted to the $\mathrm{CB}$, leading to 

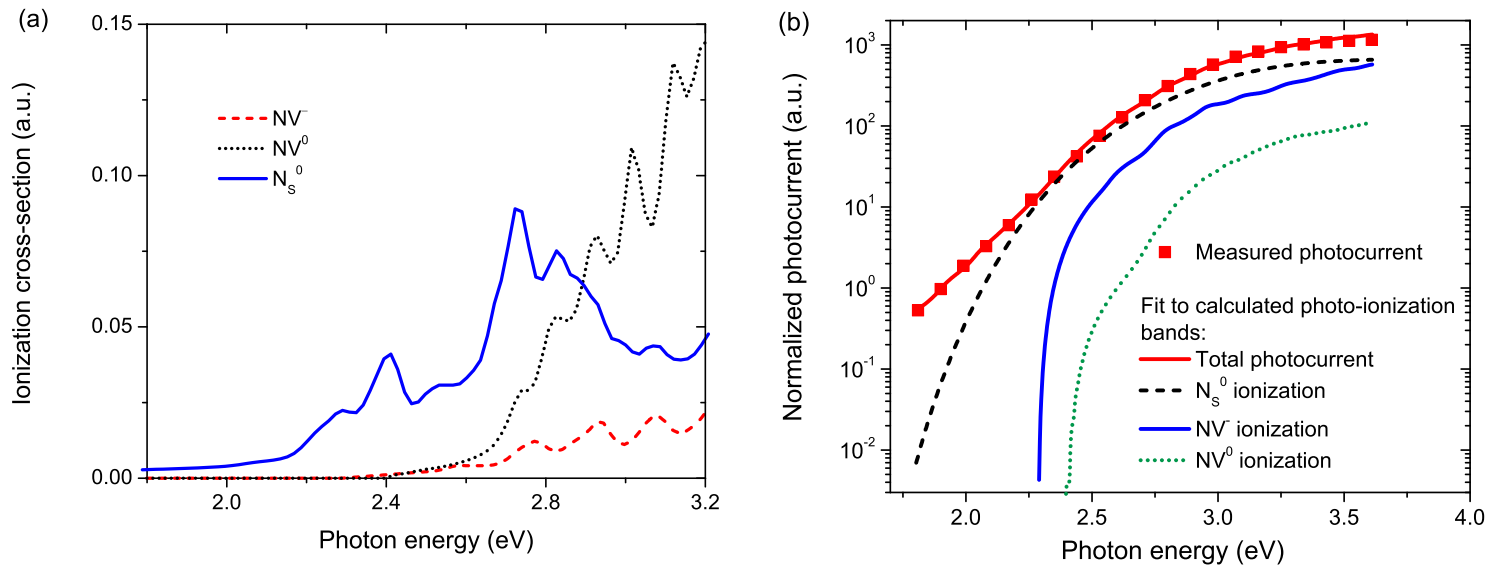

FIG. 3. (a) Calculated ionization cross section of $\mathrm{N}_{\mathrm{s}}^{0}, \mathrm{NV}^{-}$, and $\mathrm{NV}^{0}$. (b) Fitting of photocurrent measured on sample A using calculated ionization cross sections. The contributions of $\mathrm{N}_{\mathrm{s}}^{0}, \mathrm{NV}^{-}$, and $\mathrm{NV}^{0}$ ionizations to the total photocurrent are shown. In the ab initio calculation it was assumed that $\mathrm{NV}^{-}, \mathrm{NV}^{0}$, and $\mathrm{N}_{\mathrm{s}}^{0}$ ionization dominates the spectrum, with other parasitic defects contributing to a small extent to the spectrum in the low energy region (not shown).

the ionized $\mathrm{N}_{\mathrm{s}}^{+}$. Though its calculated $(+\mid 0)$ pure electronic charge transition level is at $E_{\mathrm{C}}-1.7 \mathrm{eV}\left(E_{\mathrm{C}}: \mathrm{CB}\right.$ minimum), the giant redistribution of the position of the $\mathrm{N}$ and $\mathrm{C}$ atoms in the core of the defect upon ionization of $\mathrm{N}_{\mathrm{s}}^{0}$ results in a low ionization cross section at this energy. Due to a very strong electron-phonon interaction, a photoionization band emerges in the phonon sidebands around $E_{\mathrm{C}}-2.2 \mathrm{eV}$ (see Refs. [25,26] for further discussion).

Unlike $\mathrm{N}_{\mathrm{s}}, \mathrm{NV}^{0}$ and $\mathrm{NV}^{-}$present very similar geometries, thus zero-phonon-line (ZPL) or pure electronic transitions dominate the ionization process. For $\mathrm{NV}^{-}$, the electron may be promoted from the occupied $e$ orbital to the $\mathrm{CB}$ edge. This process requires about $2.74 \mathrm{eV}$ (including the reorganization energy of atoms due to ionization), which is the ZPL energy. For $\mathrm{NV}^{0}$, a $\mathrm{ZPL}$ energy of $2.78 \mathrm{eV}$ is needed to promote an electron from the valence band (VB) edge to the empty $e$ orbital in the gap. The promotion of an electron from the VB edge to the empty $e$ orbital in $\mathrm{NV}^{-}$or from the occupied $e$ orbital in $\mathrm{NV}^{0}$ to the $\mathrm{CB}$ edge requires more than $4 \mathrm{eV}$. The calculated ionization and backionization thresholds of NV centers are within $0.04 \mathrm{eV}$ precision (within the accuracy of the method) and can therefore not be resolved in photocurrent spectra. The optical transition dipole moment is approximately three times larger for $\mathrm{NV}^{0}$ than for $\mathrm{NV}^{-}$at $2.75 \mathrm{eV}$. It should, however, be noted that the contributions of $\mathrm{NV}^{0}$ and $\mathrm{NV}^{-}$ionization to the total photocurrent also depend on their relative concentrations at a particular laser intensity.

We compared the results of these calculations with the photocurrent measurements [Fig. 3(b) for sample A, Supplemental Fig. 5 for sample E2]. In the simulation plots we set the experimental value of $\left[\mathrm{N}_{\mathrm{s}}^{0}\right]$ and fit $\left[\mathrm{NV}^{0}\right]$ and $\left[\mathrm{NV}^{-}\right]$ to the experimental data. Using only these two fitting parameters we obtained $\left[\mathrm{NV}^{-}\right] \approx 31.4 \mathrm{ppm}$ and $\left[\mathrm{NV}^{0}\right] \approx 1.0 \mathrm{ppm}$ in sample A, showing the excellent agreement of the DFT model with the results from photoluminescence measurements $\left(\left[\mathrm{NV}^{-}\right] \approx 34.0 \mathrm{ppm}\right.$ and $\left.\left[\mathrm{NV}^{0}\right] \approx 1.1 \mathrm{ppm}\right)$.

$A b$ initio calculations indicate that photons with energy above $2.7 \mathrm{eV}$ can ionize $\mathrm{NV}^{-}$to $\mathrm{NV}^{0}$, but also convert $\mathrm{NV}^{0}$ back to $\mathrm{NV}^{-}$by one-photon processes. Based on this result, we designed the dual-beam PDMR (d-PDMR) scheme, which can be described as follows [Fig. 4(a)]. Pulsed blue light (2.75 eV) directly promotes electrons from the $\mathrm{NV}^{-}$triplet ground state ${ }^{3} A_{2}$ to the $\mathrm{CB}$ (transition 1), and induces the backconversion from $\mathrm{NV}^{0}$ to $\mathrm{NV}^{-}$(transition 4). Simultaneous illumination by a cw green laser light $(2.33 \mathrm{eV})$ induces transitions from the ground state ${ }^{3} A_{2}$ to the excited state ${ }^{3} E$ (transition 2), followed by spin-selective nonradiative decay from the $| \pm 1\rangle$ spin manifold of ${ }^{3} E$ to the singlet state ${ }^{1} A_{1}$ (transition 3 ) [27]. From there, electrons fall to the metastable state ${ }^{1} E$ (220 ns lifetime) [28]. At the resonant microwave frequency $(2.87 \mathrm{GHz})$, these shelving transitions result in a temporary decrease in the occupation of the $\mathrm{NV}^{-}$ground state, and thus to a decrease in the one-photon ionization photocurrent of $\mathrm{NV}^{-}$. Here we assume that the photoionization cross section from the ${ }^{1} E$ shelving state to the $\mathrm{CB}$ is low, although ${ }^{1} E$ has been recently estimated to be located approximately $2.3 \mathrm{eV}$ below the CB [29] and could therefore theoretically be ionized by $2.75 \mathrm{eV}$ photons. However, the negative sign of magnetic resonances observed in d-PDMR [Fig. 4(b), inset] indicates that the contribution of this process to the total photocurrent is significantly lower than the contribution of direct transitions from the $\mathrm{NV}^{-}$ground state to the $\mathrm{CB}$.

The d-PDMR scheme was tested on ensembles of $\sim 10$ shallow $\mathrm{NV}^{-}$implanted in electronic grade diamond (sample TP4). As a reference, s-PDMR was measured on the same sample. At a fixed microwave power of $1 \mathrm{~W}$, a maximum s-PDMR contrast of $8.9 \pm 0.3 \%$ was obtained [Fig. 4(b), inset], three times higher than the PDMR contrast previously observed on bulk irradiated type-Ib and type-IIa diamonds [11]. Under green illumination the PDMR contrast is limited by the background photocurrent resulting from $\mathrm{N}_{\mathrm{s}}^{0}$ ionization [11]. The enhancement of the s-PDMR contrast observed here compared to previous measurements on bulk samples can be explained by the higher illumination power density (due to the two-dimensional implantation of NV centers in sample TP4 and the resulting confinement of the defects to the waist of the illumination beam, where the power density is the highest), which leads to a higher contribution of the NV two-photon ionization to the total photocurrent (see Supplemental Fig. 6). 
(a) Free

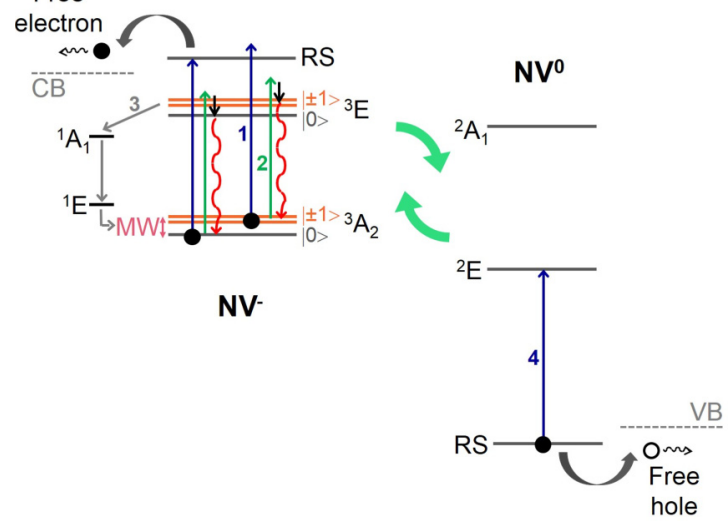

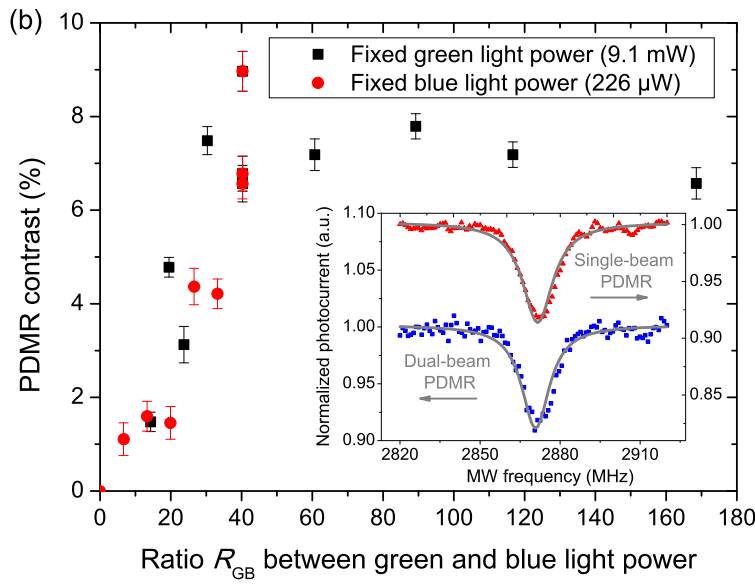

FIG. 4. (a) Schematic diagram of the d-PDMR mechanism (not to scale). Left: One-photon ionization of $\mathrm{NV}^{-}$. Right: Backconversion from $\mathrm{NV}^{0}$ to $\mathrm{NV}^{-}$. RS: Resonant state. (b) d-PDMR contrast measured as a function of the ratio $R_{\mathrm{GB}}$ (sample TP4). Error bars represent the standard errors of the fitting parameters. Inset: Comparison between d-PDMR (pulsed blue excitation, $226 \mu \mathrm{W}$; cw green excitation, $9.1 \mathrm{~mW}$ ) and s-PDMR (pulsed green excitation, $3 \mathrm{~mW}$ ) spectra measured on shallow ensembles of $10 \mathrm{NV}^{-}$, in the conditions leading to maximum MR contrast (sample TP4; microwave power, $1 \mathrm{~W}$ ). Symbols: Experimental points. Solid lines: Lorentzian fit.

To determine the conditions leading to a maximal d-PDMR contrast, at a fix microwave power $(1 \mathrm{~W})$ we varied the ratio $R_{\mathrm{GB}}$ between the green and blue light powers, controlling the relative rates of direct ionization, backconversion, and shelving transitions to the metastable state. The contrasts obtained by varying the green light power at constant blue power and the blue power at constant green power present a similar trend [Fig. 4(b)] (see note 4 in the Supplemental Material for a more detailed discussion), showing that in the range of laser power considered here and for $R_{\mathrm{GB}}<40$, the d-PDMR scheme allows an independent control of the photocurrent intensity (by the blue light power) and MR contrast (by $R_{\mathrm{GB}}$ ). A maximum d-PDMR contrast of $9.0 \pm 0.4 \%$ was obtained on sample TP4 [Fig. 4(b), inset], close to the maximum s-PDMR contrast observed on the same sample at an identical microwave power.

Although on shallow implanted $\mathrm{NV}^{-}$ensembles in electronic grade diamond the maximal MR contrasts obtained by dPDMR (under $226 \mu \mathrm{W}$ blue illumination) and s-PDMR (under $3 \mathrm{~mW}$ green illumination) are similar, the d-PDMR scheme results in a clear enhancement of the MR contrast in the case of low illumination power. Indeed, while a d-PDMR contrast of $9.0 \%$ was observed under $226 \mu \mathrm{W}$ blue illumination, the MR could not be distinguished in the photocurrent induced by green illumination of a similar power. This can be attributed to the fact that the proportion of the total photocurrent resulting from the two-photon ionization of $\mathrm{NV}^{-}$(quadratic fraction) remains very small under low green illumination power. The d-PDMR scheme should in addition lead to a higher contrast than s-PDMR in the case of samples with a high $\left[\mathrm{N}_{\mathrm{s}}^{0}\right] /\left[\mathrm{NV}^{-}\right]$ ratio, due to the lower contribution of $\mathrm{N}_{\mathrm{s}}^{0}$ ionization to the total photocurrent under blue illumination than under green illumination of similar power. Indeed, considering the green light power dependence of the photocurrent measured on type-Ib sample E2 [Supplemental Fig. 6(a)], under $4 \mathrm{~mW}$ green excitation the two-photon ionization of NV (quadratic fraction of the photocurrent) represents only $\sim 1.5 \%(0.6 \mathrm{pA})$ of the total photocurrent measured on this sample, while $a b$ initio calculations indicate that under $4 \mathrm{~mW}$ blue illumination $\sim 30 \%(0.6 \mathrm{nA})$ of the total measured photocurrent originates from the one-photon ionization of $\mathrm{NV}^{-}$(Supplemental Fig. 5).

Measurements of the photocurrent as a function of green and blue light power on sample TP4 [Supplemental Figs. 6(b) and 7(b)] show in addition that at an identical power, blue excitation induces a higher photocurrent than green excitation. For example, the photocurrent measured under 226 $\mu \mathrm{W}$ excitation on $\sim 10 \mathrm{NV}$ centers is five times higher under blue ( $800 \mathrm{fA}$ ) than under green light (165 fA). Since the ratio between the detected photocurrent and the shot noise scales as the root mean square of the photocurrent, d-PDMR should lead to a higher $\mathrm{S} / \mathrm{N}$ ratio than $\mathrm{S}-\mathrm{PDMR}$ under a similar illumination power. Based on the signal detected on $10 \mathrm{NV}$ centers, a photocurrent of $\sim 80 \mathrm{fA}$ can be expected from a single NV center under $226 \mu \mathrm{W}$ blue illumination, proving that the d-PDMR scheme could be used for the photoelectric readout of single $\mathrm{NV}^{-}$centers.

In conclusion, we performed a detailed experimental and theoretical study of NV photoionization mechanisms and developed a one-photon ionization scheme for the photoelectric readout of $\mathrm{NV}^{-}$magnetic resonances. This dual-beam PDMR scheme, which allows an independent control of the photoionization rate and MR contrast, results in a higher photoionization rate and thus a higher $\mathrm{S} / \mathrm{N}$ ratio than s-PDMR. Dual-beam PDMR leads in addition to enhanced MR contrast under low illumination power or in the case of samples with a high $\left[\mathrm{N}_{\mathrm{s}}^{0}\right] /\left[\mathrm{NV}^{-}\right]$ratio. A maximal PDMR contrast of $9.0 \%$ was obtained on ensembles of $\sim 10$ shallow $\mathrm{NV}^{-}$ centers implanted in electronic grade diamond. This robust photoelectric detection scheme could therefore represent an important step toward the photoelectric readout of single $\mathrm{NV}^{-}$spin states and be used for the construction of diamond quantum optoelectronics devices with enhanced performances.

Support from EU (FP7 Project DIADEMS, Grant No. 611143) is acknowledged. A.G. acknowledges the Lendület program of the Hungarian Academy of Sciences. The authors thank A. Jarmola and D. Budker from the Department of 
Physics of the University of California (Berkeley, California) for the preparation of the electron-irradiated type-Ib diamond.

E.B., K.B., and M.G. performed the experiments. E.B. processed the experimental data and performed the analysis. E.L. and A.G. carried out the $a b$ initio developments and calculations. J.H. wrote the software used for PDMR measurements. Y.B. prepared electrodes on type-Ib diamond samples. G.W. designed and built the blue diode laser and designed the MW antennas. J.S. prepared the proton-irradiated type-Ib diamond. K.D. and F.A. performed the diamond ion implantation. M.T. proposed the use of implanted defects, designed the electrodes, and assembled the device. M.N., M.T., and A.G. supervised the work. E.B., A.G., and M.N. wrote the manuscript. All authors discussed the results and commented on the manuscript.
[1] M. W. Doherty, N. B. Manson, P. Delaney, F. Jelezko, J. Wrachtrup, and L. C. L. Hollenberg, Phys. Rep. 528, 1 (2013).

[2] F. Jelezko and J. Wrachtrup, Phys. Status Solidi A 203, 3207 (2006).

[3] L. Childress and R. Hanson, MRS Bull. 38, 134 (2013).

[4] L. Rondin, J. P. Tetienne, T. Hingant, J. F. Roch, P. Maletinsky, and V. Jacques, Rep. Prog. Phys. 77, 056503 (2014).

[5] S. Hong, M. S. Grinolds, L. M. Pham, D. Le Sage, L. Luan, R. L. Walsworth, and A. Yacoby, MRS Bull. 38, 155 (2013).

[6] R. Schirhagl, K. Chang, M. Loretz, and C. L. Degen, Annu. Rev. Phys. Chem. 65, 83 (2014).

[7] G. Balasubramanian, A. Lazariev, S. R. Arumugam, and D. W. Duan, Curr. Opin. Chem. Biol. 20, 69 (2014).

[8] F. Shi, Q. Zhang, P. Wang, H. Sun, J. Wang, X. Rong, M. Chen, C. Ju, F. Reinhard, H. Chen, J. Wrachtrup, J. Wang, and J. Du, Science 347, 1135 (2015).

[9] P. Maletinsky, S. Hong, M. S. Grinolds, B. Hausmann, M. D. Lukin, R. L. Walsworth, M. Loncar, and A. Yacoby, Nat. Nanotechnol. 7, 320 (2012).

[10] T. Wolf, P. Neumann, K. Nakamura, H. Sumiya, T. Ohshima, J. Isoya, and J. Wrachtrup, Phys. Rev. X 5, 041001 (2015).

[11] E. Bourgeois, A. Jarmola, P. Siyushev, M. Gulka, J. Hruby, F. Jelezko, D. Budker, and M. Nesladek, Nat. Commun. 6, 8577 (2015).

[12] F. M. Hrubesch, G. Braunbeck, M. Stutzmann, F. Reinhard, and M. S. Brandt, Phys. Rev. Lett. 118, 037601 (2017).

[13] A. Dréau, M. Lesik, L. Rondin, P. Spinicelli, O. Arcizet, J.-F. Roch, and V. Jacques, Phys. Rev. B 84, 195204 (2011).

[14] See Supplemental Material at http://link.aps.org/supplemental/ 10.1103/PhysRevB.95.041402 for complementary information on material and methods, the results of photoluminescence, FTIR, and UV-vis analysis of irradiated and annealed type-Ib diamond, a fitting of the photocurrent spectrum recorded on sample E2 using $a b$ initio calculated ionization cross sections, a discussion on variations of the d-PDMR contrast with the ratio between green and blue light power, and a discussion on the green and blue light power dependence of the photocurrent measured on irradiated and annealed type-Ib diamond and implanted electronic grade type-IIa diamond.

[15] A. Gali, E. Janzén, P. Deák, G. Kresse, and E. Kaxiras, Phys. Rev. Lett. 103, 186404 (2009).

[16] P. Deák, B. Aradi, T. Frauenheim, E. Janzén, and A. Gali, Phys. Rev. B 81, 153203 (2010).

[17] J. Heyd, G. E. Scuseria, and M. Ernzerhof, J. Chem. Phys. 118, 8207 (2003).

[18] A. V. Krukau, O. A. Vydrov, A. F. Izmaylov, and G. E. Scuseria, J. Chem. Phys. 125, 224106 (2006).

[19] J. P. Perdew, K. Burke, and M. Ernzerhof, Phys. Rev. Lett. 77, 3865 (1996).

[20] M. Mrózek, J. Mlynarczyk, D. S. Rudnicki, and W. Gawlik, Appl. Phys. Lett. 107, 013505 (2015).

[21] J. Chen, S. Lourette, K. Rezai, T. Hoelzer, M. Lake, M. Nesladek, L.-S. Bouchard, P. Hemmer, and D. Budker, arXiv:1607.08354.

[22] J. Inkson, Physica C: Solid State Phys. 14, 1093 (1981).

[23] M. Nesládek, L. M. Stals, A. Stesmans, K. Iakoubovskij, G. J. Adriaenssens, J. Rosa, and M. Vaněček, Appl. Phys. Lett. 72, 3306 (1998).

[24] J. Rosa, M. Vaněček, M. Nesládek, and L. M. Stals, Diamond Relat. Mater. 8, 721 (1999).

[25] W. J. P. van Enckevort and E. H. Versteegen, J. Phys.: Condens. Matter 4, 2361 (1992).

[26] G. Thiering, E. Londero, E. Bourgeois, N. Nesladek, and A. Gali (unpublished).

[27] J.-P. Tetienne, L. Rondin, P. Spinicelli, M. Chipaux, T. Debuisschert, J.-F. Roch, and V. Jacques, New. J. Phys. 14, 103033 (2012).

[28] V. M. Acosta, A. Jarmola, E. Bauch, and D. Budker, Phys. Rev. B 82, 201202 (2010).

[29] M. L. Goldman, M. W. Doherty, A. Sipahigil, N. Y. Yao, S. D. Bennett, N. B. Manson, A. Kubanek, and M. D. Lukin, Phys. Rev. B 91, 165201 (2015). 